\title{
Understanding the Behavior of the Heliospheric Magnetic Field and the Solar Wind During the Unusual Solar Minimum Between Cycles 23 and 24
}

\author{
L. Zhao • L. Fisk
}

Received: 31 August 2010 / Accepted: 4 August 2011 / Published online: 4 October 2011

(C) Springer Science+Business Media B.V. 2011

\begin{abstract}
The properties of the heliospheric magnetic field and the solar wind were substantially different in the unusual solar minimum between Cycles 23 and 24: the magnetic-field strength was substantially reduced, as were the flow properties of the solar wind, such as the mass flux. Explanations for these changes are offered that do not require any substantial reconsideration of the general understandings of the behavior of the heliospheric magnetic field and the solar wind that were developed in the minimum of Cycle 22-23. Solar-wind composition data are used to demonstrate that there are two distinct regions of solar wind: solar wind likely to originate from the stalk of the streamer belt (the highly elongated loops that underlie the heliospheric current sheet), and solar wind from outside this region. The region outside the streamer-stalk region is noticeably larger in the minimum of Cycle 23-24; however, the increased area can account for the reduction in the heliospheric magnetic-field strength in this minimum. Thus, the total magnetic flux contained in this region is the same in the two minima. Various correlations among the solar-wind mass flux and coronal electron temperature inferred from solar-wind charge states were developed for the Cycle $22-23$ solar minimum. The data for the minimum of Cycle 23-24 suggest that the correlations still hold, and thus the basic acceleration mechanism is unchanged in this minimum.
\end{abstract}

Keywords Corona, structures · Coronal mass ejections, low coronal signatures $\cdot$ Solar cycle, observations $\cdot$ Solar wind, theory

The Sun-Earth Connection near Solar Minimum Guest Editors: M.M. Bisi, B.A. Emery, and B.J. Thompson

L. Zhao $(\bowtie) \cdot$ L. Fisk

Department of Atmospheric, Oceanic, and Space Sciences, University of Michigan, Ann Arbor, MI, USA

e-mail: Izh@umich.edu

L. Fisk

e-mail: lafisk@umich.edu

L. Zhao

NCAR/HAO, 3080 Center Green, Boulder, CO, USA

e-mail: Izh@ucar.edu 


\section{Introduction}

One of the principal discoveries of the Ulysses mission was the simplicity of the heliospheric magnetic field. A single current sheet, separating two regions of opposite polarity, is observed throughout the solar cycle (Smith et al., 1995; Balogh and Smith, 2001; Jones and Balogh, 2003). At solar minimum, the current sheet resides at low heliographic latitudes. As solar maximum approaches, the current sheet tilts to high latitudes, and eventually rotates over the poles, effectively accomplishing the reversal in the polarity of the heliospheric magnetic field.

The simplicity of the heliospheric magnetic field has had a number of important consequences for our understanding of the behavior of the open magnetic flux of the Sun, the component of the solar magnetic field that is carried outward with the solar wind and forms the heliospheric magnetic field. With a single current sheet, open magnetic flux can disconnect from the Sun only at the current sheet, and then only within the Alfvén point of the solar wind (Fisk and Schwadron, 2001). Only here can a single inverted "U"-shaped loop form that is not attached on either end to the Sun and be carried outward with the solar wind. Such an inverted loop should be devoid of electron heat flux, a so-called heat-flux dropout. However, heat-flux dropouts were reported to be rare (Lin and Kahler, 1992; Pagel, Crooker, and Larson, 2005) and so it was concluded that there would be little disconnection of open magnetic flux, and thus there must be some constant background level of open magnetic flux present in the heliosphere at all times.

There is about a factor of two increase in the open magnetic flux during the solar cycle, peaking at roughly solar maximum. This increase is attributed to the large loops that are dragged outward with coronal mass ejections (CMEs), which are more frequent during high solar activity (Gosling and Skoug, 2002). These large loops would cause the heliospheric magnetic field to increase indefinitely, and so it was concluded that a process known as interchange reconnection would occur: One leg of the large loop would reconnect with true open magnetic flux, again within the Alfvén point, thereby turning the loop into a large "S" shaped structure of open magnetic flux, which is carried outward with the solar wind, returning the heliospheric magnetic field to its background level (Gosling, Birn, and Hesse, 1995; Fisk and Schwadron, 2001; Crooker, Gosling, and Kahler, 2002). Interchange reconnection takes time and so when CMEs are frequent, the heliospheric magnetic-field strength increases. At solar minimum, when CMEs are relatively rare, the heliospheric magnetic field was expected to return to the background level. Indeed, Svalgaard and Cliver (2007) report that there was a relatively constant heliospheric magnetic field during each solar minimum prior to the minimum of Cycle $23-24$.

The interchange reconnection process appears to be a fundamental means by which to transport open magnetic flux on the Sun (Fisk and Schwadron, 2001; Fisk, 2005). An open field line that reconnects with the leg of a loop of opposite magnetic polarity, near the loop base, will be displaced to lie over the other leg of the loop. This process occurs with the large loops of CMEs, and it is reasonable to expect that it will occur with smaller coronal loops, many of which are randomly oriented. By this process, then, open magnetic flux, executing these random jumps along the solar surface, can diffuse (Fisk and Schwadron, 2001; Fisk, 2005). Since the size of the loops with which the open field lines are reconnecting can be larger than supergranules, diffusion by reconnecting with loops can be a more important transport process than diffusion by random convective motions in the photosphere.

An efficient diffusive transport mechanism of open magnetic flux on the Sun is essential and has important consequences. Differential rotation across the rigidly rotating polar coronal holes will tend to drive open flux into the surrounding closed-field regions (Fisk, 1996; 
Fisk, Zurbuchen, and Schwadron, 1999b). Yet, as argued above, the open flux does not appear to disconnect at the current sheet. The result is that open flux needs to be transported efficiently through the surrounding closed-field region, by random reconnections with the loops (Fisk and Zurbuchen, 2006). A continuous flow pattern of open flux will occur, driven by the differential rotation across the polar coronal holes at solar minimum, and continued through closed-field regions by reconnection with coronal loops. At solar maximum, when the polar coronal holes are not well-established, it is still necessary to transport open flux by diffusion due to reconnection with loops, to accomplish the rotation of the current sheet (Fisk and Schwadron, 2001).

The mechanism of open magnetic flux reconnecting with coronal loops will inherently release material from the loop, and may supply the mass to the solar wind. We thus have a straightforward explanation for the compositional differences between fast and slow solar wind. The elemental composition of the slower solar wind, as compared with the fast wind from coronal holes, exhibits enhancements in elements with low first ionization potential (FIP), and relatively high coronal electron temperatures as inferred from solar-wind charge states (Zurbuchen et al., 2000). The composition of the slower solar wind closely resembles that of the large coronal loops on the quiet Sun, outside of coronal holes (Feldman, Landi, and Schwadron, 2005). All this is understandable by noting that differential rotation across the polar coronal holes will drive open flux into closed-field regions, where its continuous transport requires reconnection with the large coronal loops on the quiet Sun outside of coronal holes. The reconnection releases the loop material, resulting in the slower solar wind (Fisk, Zurbuchen, and Schwadron, 1999b).

The reconnection of open magnetic flux with coronal loops should be an important process in the acceleration of the solar wind. In addition to the mass provided to the solar wind by the release of material from the loop by reconnection, the reconnection process will substantially displace the open field line, and disturb and provide energy into the overlying corona, which when dissipated can provide the energy that will accelerate the solar wind. Fisk, Schwadron, and Zurbuchen (1999a) and Fisk (2003) introduced a new class of solarwind theories in which the mass flux of the solar wind is determined by the release of material from loops by reconnection and the deposition of energy to accelerate the solar wind is determined by the displacement and subsequent relaxation to equilibrium of open magnetic flux in the overlying corona (see also Schwadron and McComas, 2003; Schwadron, McComas, and DeForest, 2006). This class of theory provides a natural explanation for the observed anti-correlation between solar-wind speed and the coronal electron temperature inferred from solar-wind charge states (Gloeckler, Zurbuchen, and Geiss, 2003).

Thus, as we entered the Cycle 23-24 solar minimum, we had certain expectations as to what would occur. We expected that the heliospheric current sheet would return to lie near the equatorial plane and that the strength of the heliospheric magnetic field would return to the constant background level of previous solar minima. It became obvious, however, in 2006 that this was not to be the case. The strength of the heliospheric magnetic field fell precipitously to a level never before observed when there have been adequate space observations, reaching a level 30\% below the level of previous minima (Smith and Balogh, 2008). Also, the current sheet has remained relatively tilted, and some large coronal holes extend down to low heliographic latitudes (i.e., in Carrington Rotation (CR) 2032), with more isolated equatorial coronal holes showing up later on (Abramenko et al., 2010).

These changes in the heliospheric magnetic field were accompanied by unprecedented changes in the basic solar-wind flow parameters. The proton mass flux and the ram pressure of the solar wind were decreased by $20 \%$ and $22 \%$, respectively (McComas et al., 2008), and the solar-wind charge states, and thus the coronal electron temperature decreased. All 


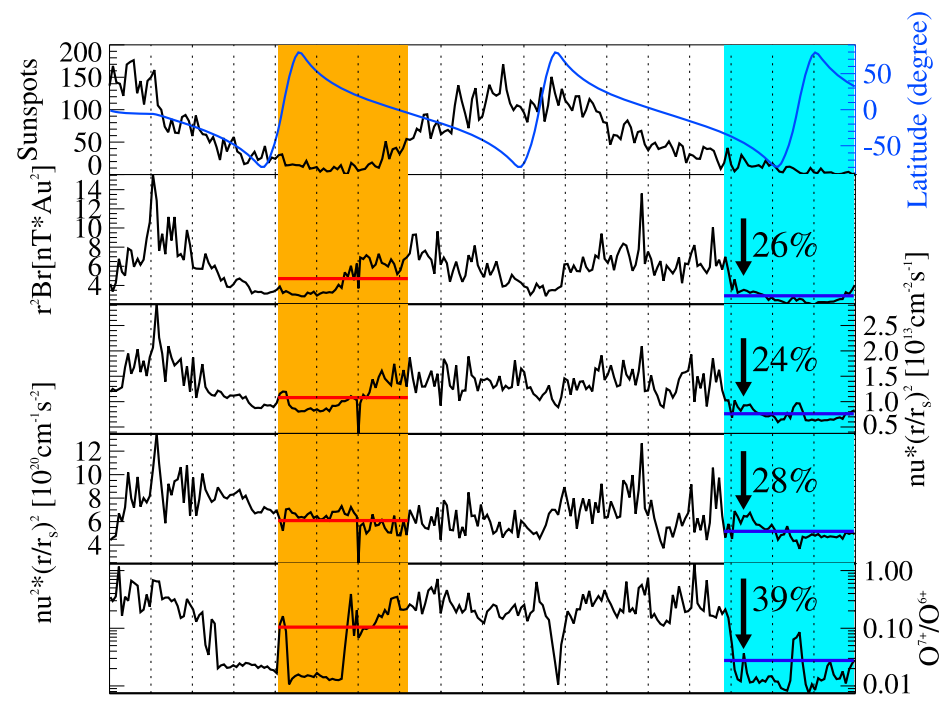

1991199319951997199920012003200520072009

Year

Figure 1 Monthly sunspot number (black), Ulysses heliographic latitudes (blue), normalized radial component of the heliospheric magnetic field $\left[B_{r} r^{2}\right]$, solar-wind mass flux [ $\left.\rho u r^{2}\right]$, solar-wind ram pressure $\left[\rho u^{2} r^{2}\right]$, and charge-state ratio of $\mathrm{O}^{7+} / \mathrm{O}^{6+}$, as observed by Ulysses from 1991 to 2009 . The Cycle $22-23$ solar minimum and the minimum of Cycle 23-24 are highlighted by yellow and blue background colors, respectively. The vertical dotted lines mark the beginnings of every year. Every data point shown in the plots is the average value for each Carrington rotation. The horizontal red (blue) lines mark the average values in the Cycle $22-23$ (Cycle 23-24) solar minimum, their decreasing percentiles are indicated with downward arrows.

of these parameters (as observed by Ulysses) - the heliospheric magnetic-field strength, the mass flux and ram pressure of the solar wind, and the solar-wind charge states - are shown in Figure 1.

The purpose of this article is to examine what alterations are required to our understanding of the behavior of the heliospheric magnetic field and the solar wind, which were developed in the previous cycle, to account for the observed unusual behavior in the Cycle $23-24$ solar minimum. The reduction in the heliospheric magnetic-field strength could be due simply to an inaccurate estimate of the number of CMEs present in the heliosphere in previous solar minima (Owens et al., 2008). We had assumed that in previous minima there would be little magnetic flux in the heliosphere due to CMEs, and the observed heliospheric magnetic field would be at the constant background level. If this were not correct, and there was still magnetic flux associated with CMEs present, then the actual background level of open magnetic flux is lower. Then, in the Cycle 23-24 solar minimum, with its lower level of activity, there may be fewer CMEs, and we are now approaching the background level. This explanation does not require any alterations in the basic concepts for the behavior of the heliospheric magnetic field that have been developed, only a lower actual background level of open magnetic flux.

In this article, we offer an alternative explanation for the reduction in the strength of the open magnetic flux in the Cycle 23-24 solar minimum. We introduce a somewhat more complex structure for the heliospheric magnetic field. We argue that there is a separate component of the heliospheric magnetic field that immediately surrounds the heliospheric 
current sheet, and which we suggest traces its origin to the extended coronal loops that underlie the current sheet, which we refer to as the streamer-stalk region. The solar wind in this region surrounding the current sheet is clearly different in composition; it has distinctly higher charge states, indicating a source in hotter loops, and is quite slow. We demonstrate that the width of the streamer-stalk solar wind, relative to the current sheet, is demonstrably narrower in the Cycle 23-24 solar minimum compared to the previous minimum. Consequently, the region outside the streamer-stalk region is demonstrably larger. We then show that the total magnetic flux in the region outside the streamer-stalk region-the product of the increased solid angle and the observed reduced magnetic field strength - is the same in the Cycle 23-24 and Cycle 22-23 solar minima. In other words, the total magnetic flux in the background level of open flux, in the region outside the stream-stalk region, is constant from cycle to cycle. As we will demonstrate, this modified understanding of the behavior of the heliospheric magnetic field requires only minor alterations to the basic concepts for the behavior of the heliospheric magnetic field that have been developed.

We have a more unqualified success with regard to the solar-wind models that were developed in the previous cycle. We find that the formulae that were developed to account for the mass flux of the solar wind and to explain the anti-correlation between solar-wind flow speed and coronal electron temperature all hold in the minimum of Cycle 23-24, without alteration.

We begin with our explanation for the behavior of the heliospheric magnetic field during the Cycle 23-24 unusual solar minimum, and its consequences. We then show that our basic solar-wind formulae appear to remain valid, and in Concluding Remarks we summarize these results and consider additional research that still needs to be pursued.

\section{The Behavior of the Heliospheric Magnetic Field}

\subsection{Two Types of Solar Wind}

The streamer region is the large, extended loops under the heliospheric current sheet. It can extend to a few solar radii. Since the streamer-belt loops are hotter, the solar wind coming from the stalks of the streamer belt should exhibit substantially hotter temperatures. Particularly their ion compositions (such as the $\mathrm{O}^{7+} / \mathrm{O}^{6+}$ and $\mathrm{C}^{6+} / \mathrm{C}^{5+}$ ratios) that freeze-in very quickly and remain the same during propagation through the heliosphere should exhibit relatively high values (Geiss, Gloeckler, and von Steiger, 1995; von Steiger et al., 2000). Therefore, we assume that streamer wind can be determined by their relatively high chargestate ratio, like $\mathrm{O}^{7+} / \mathrm{O}^{6+}$. We use the in-situ observations to show that the band of the highest $\mathrm{O}^{7+} / \mathrm{O}^{6+}$ ratio wind, larger than 0.145 (Zhao, Zurbuchen, and Fisk, 2009; Zurbuchen et al., 2002), comes from the stalks of the streamer belt, the largest extended loops that underlie the heliospheric current sheet. Thus, we have three types of solar wind based on their different origins: streamer-stalk wind, non-streamer-stalk wind (some of which comes from coronal holes and some of which is still low-speed wind from loops at higher latitudes), and transient interplanetary coronal mass ejections (ICMEs).

We repeat the analysis of Zhao, Zurbuchen, and Fisk (2009) to determine the three types of solar wind, using the criteria as shown in Table 1. In Figure 2, we show the fractions of these three types of wind in the Ulysess 18-year observations along with the monthly sunspot number. The average streamer-stalk wind (orange) contributes $27 \%$ to the heliosphere and is a very variable portion; the non-streamer-stalk wind (green) covers $63 \%$ of the time and can be considered as the majority of the heliosphere; and ICMEs (yellow) participate about $10 \%$ 
Figure 2 Monthly sunspot number (top) and three solar-wind components (bottom) during 1991 - 2009: ICMEs (yellow), non-streamer wind (green) and streamer wind (orange).

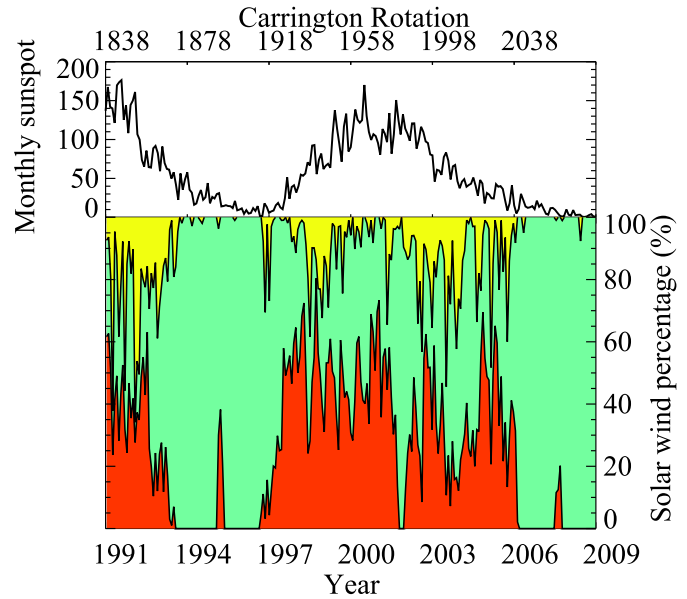

Table 1 In-situ signatures of three types of solar wind.

\begin{tabular}{llll}
\hline & Signature & $V_{\text {SW }}$ Relationship & Criterion For \\
\hline 1 & $\mathrm{O}^{7+} / \mathrm{O}^{6+}$ & $\mathrm{O}^{7+} / \mathrm{O}^{6+} \geq 6.008 \exp \left(-0.00578 V_{\mathrm{sw}}\right)$ & ICMEs \\
2 & $\mathrm{O}^{7+} / \mathrm{O}^{6+}$ & $0.145<\mathrm{O}^{7+} / \mathrm{O}^{6+}<6.008 \exp \left(-0.00578 V_{\mathrm{SW}}\right)$ & Streamer-stalk wind \\
3 & $\mathrm{O}^{7+} / \mathrm{O}^{6+}$ & $\mathrm{O}^{7+} / \mathrm{O}^{6+} \leq 0.145$ & Non-streamer-stalk wind \\
\hline
\end{tabular}

of the time, and their occurrence rate is approximately proportional to the sunspot number and can also indicate the level of solar activity.

The wind from outside of the streamer-stalk region consists of two main groups. The first group is the wind that originates exclusively from low-temperature coronal holes; those winds have high proton speed $\left(V>600 \mathrm{~km} \mathrm{~s}^{-1}\right)$, are relatively stable and normally are distributed at high latitudes in solar minimum; the other group is the low-speed wind $\left(V<600 \mathrm{~km} \mathrm{~s}^{-1}\right)$ from other lower-temperature regions outside of coronal holes; those winds are distributed in broader regions from low to mid latitudes (Tokumaru et al., 2009).

In previous solar minima, low-latitude coronal holes were uncommon (Phillips et al., 1995). However, this is not the case during the Cycle 23-24 solar minimum. With the aid of the $195 \AA$ images from the Extreme ultraviolet Imaging Telescope (EIT) on SOHO, we can construct a general view of the coronal holes during the two solar minima. Figure 3, CR 2033 (8 August 2005 - 4 September 2005) is an example; the coronal-hole regions are shown by black pixels. By marking each of the black-coronal-hole pixels by white and calculating the area covered by those pixels, we find that compared with the previous solar minimum (CR 1911-1941, 28 June 1996-22 October 1998), the area of the coronal holes at low latitude $\left(<45^{\circ}\right)$ increases dramatically in the minimum of Cycle 23-24 (CR 2025 - 2055, 2 January 2005-27 April 2007), by almost 380\% (Figure 4).

Because the EUV emission is very sensitive to temperature and density, the size of the dark regions in the images is very wavelength dependent. The method we use to estimate the coronal holes here is thus only approximate. Based on the single-wavelength measurement, our results may not provide an accurate evaluation of the exact area and position of the coronal holes, but are sufficiently reliable to compare the relative difference between the two solar minima. 


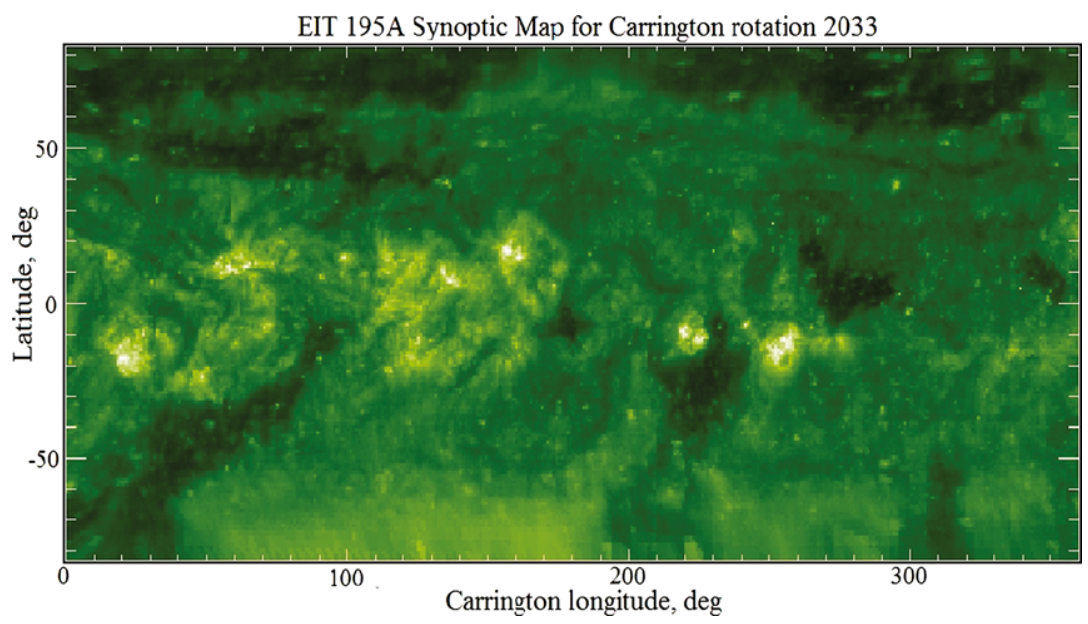

Figure 3 SOHO Extreme ultraviolet Imaging Telescope (EIT) 195 Å images at CR 2033.

Figure 4 Variation of the coronal-hole area at low latitude $\left(<45^{\circ}\right)$. Highlighted time periods are close to the minimum of Cycle 22-23 and the Cycle 23-24 solar minimum: CR 1911 - 1941 (yellow) and CR 2025 - 2055 (blue).

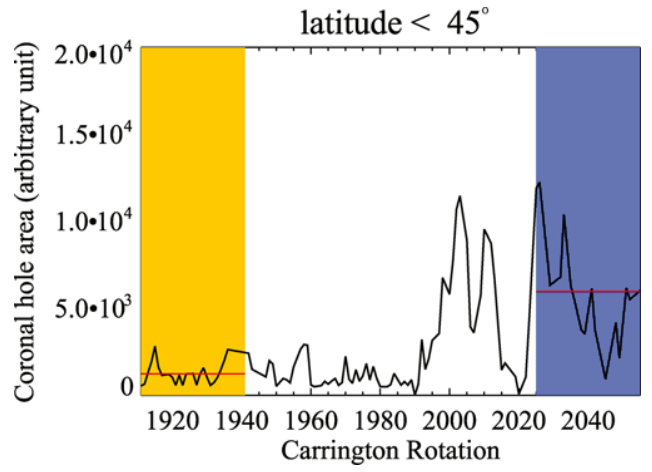

The low-latitude coronal holes still occured until the end of 2008 when they started to close down and finally disappeared in 2009 (de Toma, 2010). Besides the fact that the lowlatitude coronal holes extend to the ecliptic plane, the polar coronal holes shrink as shown by the SOHO/EIT $171 \AA, 195 \AA$, and $304 \AA$ full-disk images: the sizes of the polar coronal holes at both poles are smaller in the minimum of Cycle 23-24 than the minimum of Cycle $22-23$ (Kirk et al., 2009).

\subsection{The Width of the Streamer-Stalk Region}

To have an insightful view of where the three types of wind originate from the solar corona, we map all of the in-situ Ulysses measurements back to the source surface used for the PFSS (Potential Field Source Surface) model, at 2.5 solar radii, following the same mapping technique introduced by Zhao, Zurbuchen, and Fisk (2009). Figure 5 is an example from CR 2060 (14 August 2007 - 10 September 2007, corresponding to Ulysses' fast latitude scan). In this figure, the background contours show the magnetic polarities from the PFSS model: the dashed (solid) lines represent the inward (outward) magnetic field and the purple line is the current sheet. The black line in the middle of the color band is the trajectory of Ulysses, the color bars above the black line indicate the two solar-wind types (non-streamer wind 


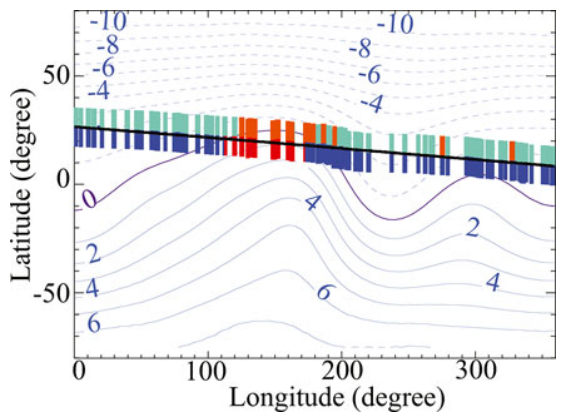

Figure 5 Origin of three types of solar wind in CR 2060. Background contours show the magnetic polarities from the PFSS model: the dashed (solid) lines represent the inward (outward) magnetic field and the purple line is the current sheet. The black line in the middle of the color band is the trajectory of Ulysses, the color bars above the black line indicate the two solar-wind types (non-streamer wind in green and streamer wind in orange) and the color bars under the black line show observed magnetic polarities (inward in blue and outward in red).

in green and streamer wind in orange), and the color bars under the black line show the observed magnetic polarities (inward in blue and outward in red). As expected, Ulysses observes some streamer-stalk wind when it crosses the current sheet. Also, the polarities of the observed magnetic field match the PFSS model result very well. Based on these maps, we can calculate the normal distance from each of the "foot points" of the solar wind to the local current sheet. These normal distances are portions of great-circle arcs and can be expressed as an angle relative to the current sheet.

From these mapped results for all of the Carrington rotations (such as CR 2060 shown in Figure 5), we can compare the polarities of the observed magnetic field with the PFSS results. We have used observations from both ACE at $1 \mathrm{AU}$, and from Ulysses for this purpose. The comparison between the background PFSS contours and our observed polarities of the field shows that in many of the Carrington rotations, the observed polarities from ACE and the current-sheet positions found by our technique are consistent with the PFSS results. This consistency indicates that the PFSS results around the equatorial plane are reliable and that our data-analysis technique works very well. However, for the Ulysses observations, the consistency between the observed magnetic polarity and the PFSS results are not always acceptable. This deviation between observations and the PFSS is likely due to the limitation of the PFSS model at the high heliographic latitudes, where the observations of the solar magnetic field are very limited. However, since our analysis focuses on the solar minima, when the heliospheric current sheet is less tilted and remains at low latitudes, these deviations between the observations and the PFSS model are not important.

The statistic results of all of the normal distances from the solar-wind foot points to the local current sheet in the two successive solar minima as observed by Ulysses are shown in Figure 6. During the Cycle 22-23 solar minimum (25 January 1995-14 March 1998, CR 1892-1933), most (>95\%) of the streamer-stalk wind distributes around the current sheet and within $25^{\circ}$ on each side. Note that focusing only on the period when the sunspot number is at the lowest value, we find the Cycle $22-23$ solar minimum is even shorter, about 2.5 years, from 1 January $1995-11$ July 1997 (Figure 1). In such a short time interval, the available streamer-stalk solar-wind data are limited because Ulysses is not always at low latitude. To use all of the possible streamer-stalk solar-wind data observed by Ulysses, we do not exclude the period when Ulysses is at high latitude, but carefully examine the Ulysses 
Figure 6 Ulysses observation: probability densities of the normal distances from the source of streamer-stalk wind to the local heliospheric current sheet on the 2.5 solar radii surface during the Cycle 22-23 solar minimum (25 January 1995 - 14 March 1998, CR 1892-1933) (solid line) and during the Cycle 23-24 solar minimum (29 October 2005-16 December 2008, CR 2036-2077) (dotted line).

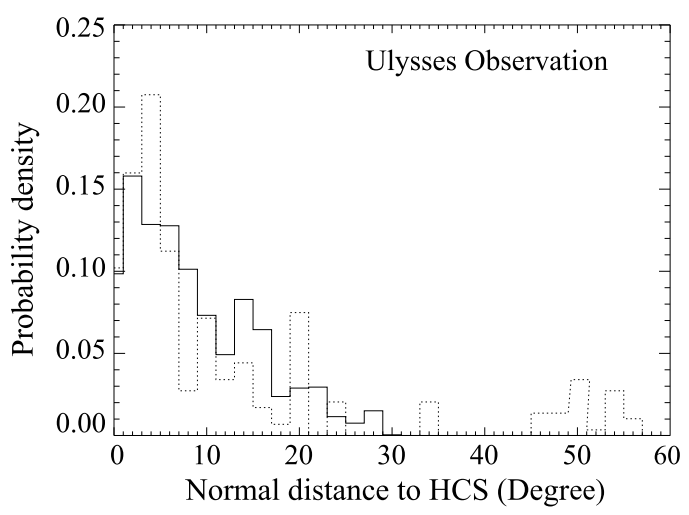

observation in several time intervals within this solar minimum. We examine the streamerstalk width in three individual periods: 1995, 1996 and 1995-1996. We find that if we set $80 \%$ as the criterion, there will be more than $80 \%$ of the streamer-stalk wind distributed within $24^{\circ}(1995), 22^{\circ}(1996)$, and $22^{\circ}(1995-1996)$ around the current sheet in the three time intervals, respectively. If we apply $90 \%$ as the criterion, the estimated half width will be even larger. Therefore, we conclude that the half width of the streamer-stalk region in $22 / 23$ minimum is $\approx 25^{\circ}$, which is consistent with the previous publications (Richardson and Paularena, 1997; Phillips et al., 1995).

In contrast, in the minimum of Cycle 23-24 (29 October 2005-16 December 2008, CR 2036-2077), the majority of the streamer-stalk wind comes from a band around the current sheet within about $10^{\circ}$ on each side, or in total the streamer-stalk is $15^{\circ}-20^{\circ}$ wide (Figure 6). The significantly different distribution of the streamer-stalk wind relative to the current sheet suggests that besides all of the parameters shown in Figure 1 that are different between the two minima, the solar-wind structure in the heliosphere is also different, and all of these changes can be related to each other. Note that we still have some high $\mathrm{O}^{7+} / \mathrm{O}^{6+}$ ratio (high electron temperature) wind that originates somewhat farther away from the current sheet during the minimum of Cycle 23-24. There are three possible explanations for the distribution of these high electron-temperature winds: First, the high-temperature wind can be produced by the reconnection between hotter, large coronal loops and the open field lying outside of the streamer-stalk region. Second, some of the high $\mathrm{O}^{7+} / \mathrm{O}^{6+}$ ratio wind far from the heliospheric current sheet may be due to the uncertainty of the simulated current sheet determined by the PFSS model. Third, Ulysses' large heliocentric distance may also cause some errors in the trajectory-mapping process.

Unlike Ulysses, ACE provides us with in-situ observation near 1 AU. The current-sheet crossings observed by ACE have been proven to be more accurate and more consistent with the PFSS model than Ulysses (Zhao, Zurbuchen, and Fisk, 2009). Therefore, we also used ACE observation to examine the width of the streamer-stalk region. We found during the Cycle 23-24 solar minimum, about $90 \%$ of the streamer-stalk wind as observed by ACE is from a region of $10^{\circ}$ around the current sheet (Zhao and Fisk, 2010) (Figure 7). In combining both Ulysses and ACE observations, we find the majority (80\%) of the streamer-stalk wind originates from a band about $10^{\circ}$ around the current sheet (Figure 8). Note that ACE data are not available for the minimum of Cycle 22-23, so only Ulysses data can be used for a similar analysis in that minimum, and hence are used in this article.

We need to emphasize that the signature that we use to identify our streamer-stalk wind is the $\mathrm{O}^{7+} / \mathrm{O}^{6+}$ ratio, or the electron temperature inferred from the ratio, and not 

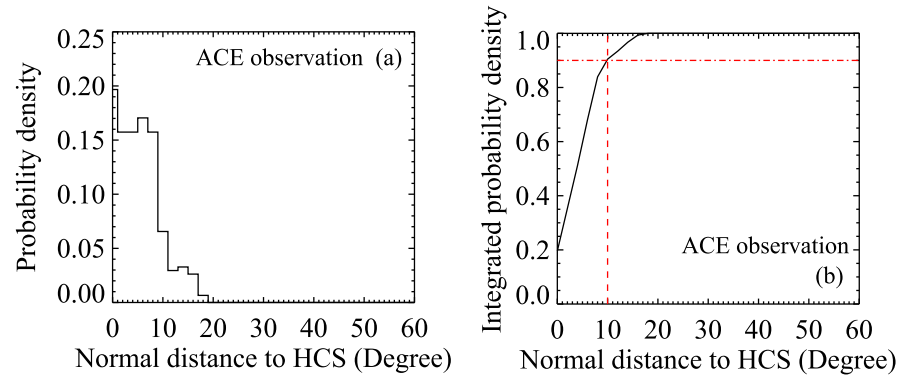

Figure 7 (a) Probability density of the normal distances of the streamer-stalk wind to the current sheet in the minimum of Cycle 23-24 from ACE observations. (b) Integration of the probability density performed from where the normal distance is $0^{\circ}$ to $60^{\circ}$ (along the $x$-axis from left to the right). The horizontal dotted line marks where the integrated probability density is $90 \%$ and the vertical dotted line marks where the normal distance is $\approx 10^{\circ}$.
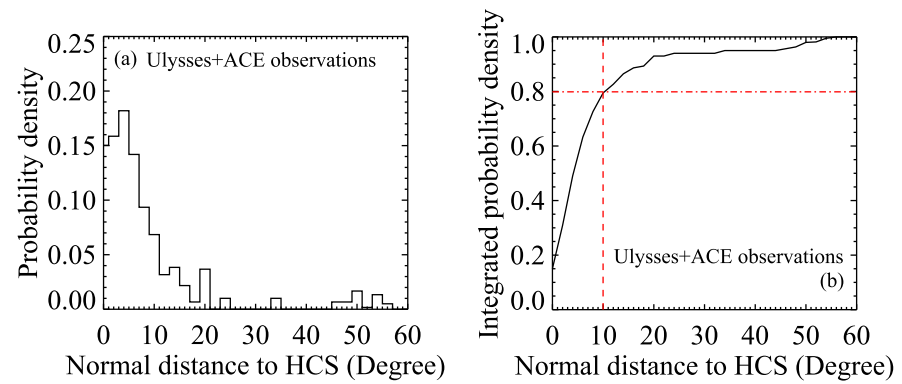

Figure 8 (a) Probability density of the normal distances of the streamer-stalk wind to the current sheet in the minimum of Cycle 23-24 from the combination of ACE and Ulysses observation. (b) Integration of the probability density performed from where the normal distance is $0^{\circ}$ to $60^{\circ}$ (along the $x$-axis from left to the right). The horizontal dotted line marks where the integrated probability density is $80 \%$ and the vertical dotted line marks where the normal distance is $\approx 10^{\circ}$.

the solar-wind speed, which distinguishes this effort from other studies (Miyake et al., 1989; Kojima and Kakinuma, 1987). It is also important to note that the streamer-stalk wind discussed here is not the entire low-speed solar wind. Rather, it is the slowest wind from the streamer-stalk region around the current sheet. Using a similar procedure and based on Ulysses observation, we can also examine the distribution of the low-speed wind relative to the heliospheric current sheet. We find the low-speed wind $\left(V<600 \mathrm{~km} \mathrm{~s}^{-1}\right)$ is distributed in a similar region around the heliospheric current sheet as the streamer-stalk wind in the Cycle $22-23$ minimum, but in a wider region in this solar minimum (Figure 9). Interestingly, the latitudinal distribution of low-speed wind in the Cycle 22-23 solar minima has also been reported wider (Neugebauer et al., 1998; Crooker et al., 1996) than the width of the streamer-stalk wind as we calculated in the minimum of Cycle 23-24; while in the Cycle 21-22 minimum, the slow solar-wind band is much narrower than the value in the minimum of Cycle 22-23 (Miyake et al., 1989; Kojima and Kakinuma, 1987).

The broader, slow solar-wind region has also been reported by Tokumaru et al. (2009). In the wider, slow solar-wind source region, the mechanism for generating the slow wind is the same as in the streamer-stalk and in coronal-hole regions for the fast wind (Fisk, Schwadron, and Zurbuchen, 1998; Fisk, Zurbuchen, and Schwadron, 1999b; Fisk, 2003): 
Figure 9 Ulysses observation: probability densities of the normal distances from the source of slow wind $\left(V<600 \mathrm{~km} \mathrm{~s}^{-1}\right)$ to the local heliospheric current sheet on a 2.5 solar radii surface in the Cycle 22-23 solar minimum (25 January 1995 - 14 March 1998, CR 1892-1933) (solid line) and the minimum of Cycle 23-24 (29 October 2005-16 December 2008, CR 2036-2077) (dotted line).

Figure 10 Histograms of the non-streamer wind data in the Cycle 22-23 solar minimum (25 January 1995-14 March 1998, CR 1892-1933, in solid line) and in the Cycle 23-24 solar minimum (29 October 2005- 16 December 2008, CR 2036-2077, in dashed line) from Ulysses.
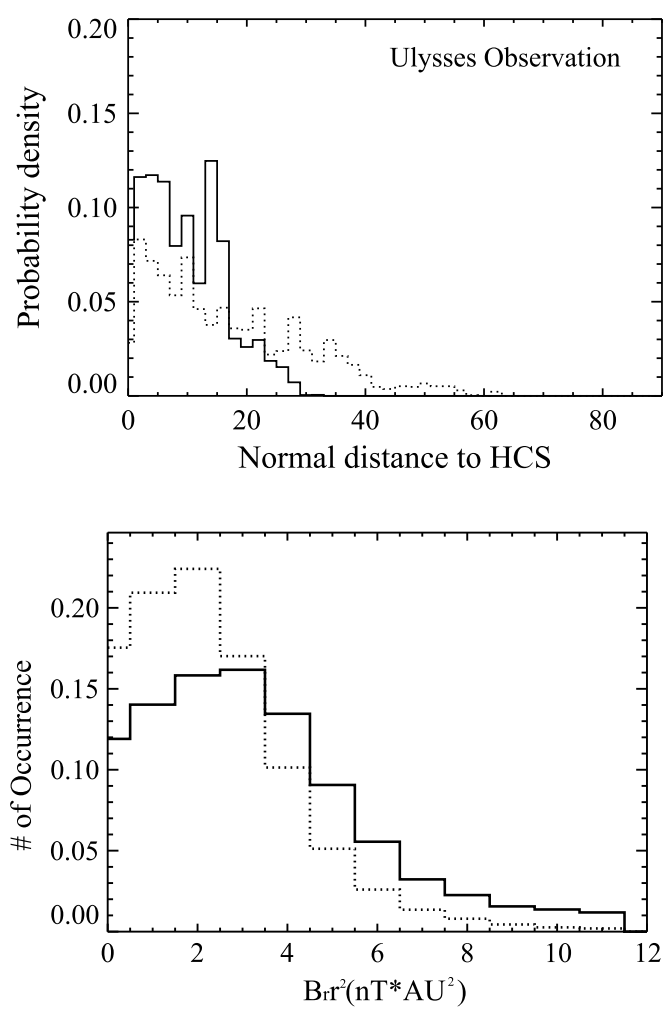

The open magnetic flux is transported by reconnection with loops. This process inherently releases material into the corona, and energy, which can be used to accelerate the solar wind.

\subsection{The Total Magnetic Flux}

We conclude in the previous section that in the minimum of Cycle 23-24 the streamerstalk region is narrower, and, as a consequence, the area outside the streamer stalk must be larger than in the Cycle 22-23 minimum. Consider, then, how the total open magnetic flux contained in the region outside the streamer-stalk region varies between the two solar minima.

The total amount of the open magnetic flux is the product of the area or solid angle $[\sigma]$ occupied by non-streamer-stalk region and the magnetic strength $\left[B_{r} r^{2}\right]$ in that region. From Table 2, the half width of the streamer belt in the Cycle $22-23$ minimum is about $25^{\circ}$, and during the minimum of Cycle $23-24$ it is reduced to $10^{\circ}$. If we assume that the solid angle covered by the non-streamer region in the minimum of Cycle $22-23$ is one, then in the minimum of Cycle $23-24$ this solid angle increases to 1.43. Also, In Figure 10, we show the histograms of the magnetic-field strength $\left[B_{r} r^{2}\right]$ in the region outside of the streamerstalk region during the minimum of Cycle 22-23 (solid line) and during the minimum of Cycle 23-24 (dotted line). The average value of $B_{r} r^{2}$ in the minimum of Cycle 22-23 is $4.3 \mathrm{nT} \mathrm{AU}^{2}$, while in the later minimum of Cycle $23-24$ this value goes down to $2.93 \mathrm{nT}$. $\mathrm{AU}^{2}$, decreasing by $31.8 \%$. Thus, the products of $\sigma$ and $B_{r} r^{2}$ in these two solar minima remain the same (Table 2). We therefore conclude that the total amount of open magnetic flux in the region outside of the streamer region remains the same in the two minima. 
Table 2 Total amount of magnetic flux outside of streamer stalk.

\begin{tabular}{lllll}
\hline & $\begin{array}{l}\text { Streamer half- } \\
\text { width (degrees) }\end{array}$ & $\begin{array}{l}\text { Non-streamer-stalk } \\
\text { region solid angle }\end{array}$ & $B_{r} r^{2}$ & $\begin{array}{l}\text { Total magnetic } \\
\text { flux }\end{array}$ \\
\hline $22-23$ minimum & 25 & 1 & 1 & 1 \\
$23-24$ minimum & 10 & 1.43 & 0.7 & $\approx 1$ \\
\hline
\end{tabular}

The conservation of the open magnetic flux in the region outside of the streamer stalk is a very robust result that is not sensitive to the time period we choose. For example, in a pair of different time periods, 7 August 1993 - 19 October 1995 (approaching 22-23 minimum, during Ulysses' first orbit), and 1 January 2006-13 March 2008 (covering the latest minimum during Ulysses' third orbit), Ulysses has the exactly same latitudinal scans, which can allow us to compare the streamer-stalk wind and magnetic flux without a latitudinal bias. We find the magnetic strength decreases by $24 \%$ in the minimum of Cycle $23-24$ compared to the period close to the minimum of Cycle $22-23$. The streamer-stalk region is about $15^{\circ}$ and $44^{\circ}$ around the heliospheric current sheet in the period close to the Cycle 23-24 and $22-23$ minima, respectively. These differences indicate that the total magnetic flux in the region outside of the streamer stalk in the later period of the minimum of Cycle 23-24 is about 1.05 times (not significantly different than 1) compared to the period close to the minimum of Cycle $22-23$.

The Ulysses' heliocentric distance varies from 1.5 AU to 5.0 AU in the Cycle 22-23 solar minimum and from 1.5 AU to 4.0 AU in the minimum of Cycle 23-24. These relatively large heliocentric distances might have some influence on the accuracy of our mapping technique, and therefore can broaden the estimated streamer-stalk width. Since Ulysses' heliocentric distance is comparable in the two solar minima, we think that if such an influence exists, it will be very similar in the two solar minima, and we can approximate its implication. For example, if both the streamer-stalk wind in the two solar minima are broadened by $5^{\circ}$ due to Ulysses' large heliocentric distance, the areas of the non-streamer-stalk region will decrease by $13 \%$ and $11 \%$ in the Cycle $22-23$ and Cycle $23-24$ minima, respectively. In addition, we know that the open magnetic field $\left[B_{r} r^{2}\right]$ is independent on latitude, i.e., it will remain the same regardless of how much the streamer-stalk region changes (Smith and Balogh, 2008). Thus, the ratio of the total magnetic flux in the non-streamer-stalk region in the two minima will remain approximately one $(87 \% / 89 \%=98 \% \approx 1)$. Therefore, Ulysses' relatively large heliocentric distance will not cause a significant change to our conclusion.

Note that in our analysis we are only interested in the non-transient solar wind, and we have ruled out the ICMEs; thus, the total open magnetic flux that we calculate is the background level at solar minimum without impact from ICMEs.

\subsection{Implications of the Conservation of Total Magnetic Flux}

The streamer-stalk wind is a separate component of solar wind; its composition, speed, temperature, variability, and spatial distribution are all different from the other solar-wind components. The conservation of total magnetic flux in the region outside of the streamerstalk region is a natural consequence of the presence of a separate component of the solar wind surrounding the heliospheric current sheet. Open magnetic flux can disconnect only at the heliospheric current sheet and then only within the Alfvén point (Fisk and Schwadron, 2001). If the open magnetic flux in the region outside of the streamer stalk is unable to 

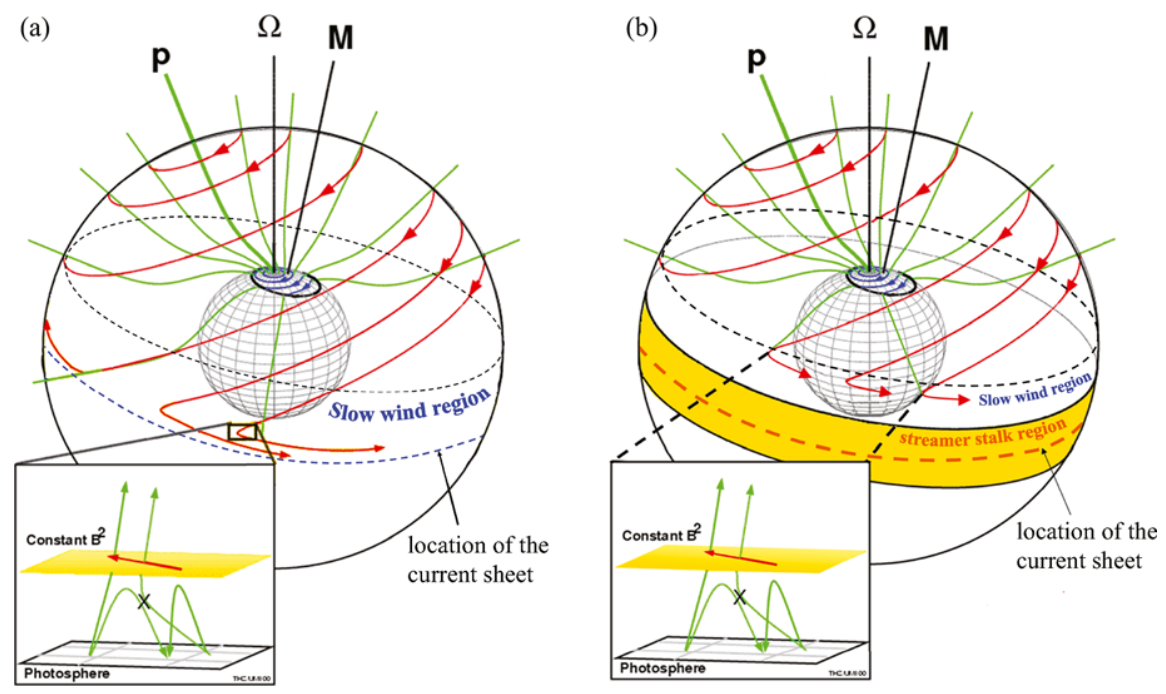

Figure 11 (a) An illustration of the motions of the magnetic field on the Sun in the frame corotating with the equatorial rotation rate (Fisk, 1996; Fisk, Zurbuchen, and Schwadron, 1999b; Fisk and Schwadron, 2001). The $M$-axis is the axis of symmetry for the expansion of the magnetic field from a polar coronal hole. The $\Omega$-axis is the solar rotation axis. $P$ marks one of the open lines (green) that connects to the Pole. The curves with arrows (red) are the trajectories of the open lines. (b) The open lines reconnect and diffuse outside the streamer-stalk region, which is marked in yellow (after Zhao and Fisk, 2010).

penetrate into the heliospheric current sheet then no disconnection is possible, and the background level of open magnetic flux in this region should remain constant throughout the solar cycle.

In a series of articles, Fisk and colleagues developed a model for the global transport of open magnetic flux on the Sun, which is illustrated in Figure 11a (Fisk, 1996, 2005; Fisk and Schwadron, 2001; Fisk, Zurbuchen, and Schwadron, 1999b). Differential rotation drives the open flux across the polar coronal hole and then into closedfield regions where open flux does not disconnect at the current sheet, but rather the flow patterns turn as shown. The process by which the magnetic field flows through the closed-field region is reconnection with coronal loops, with a resulting random jump in the foot points of the open flux and the open flux diffusing through the closed-field region. This picture now needs to be revised, as shown in Figure 11b. The open magnetic flux in regions outside the streamer-stalk region is unable to penetrate into this region. Thus, disconnection of this component of open flux, which must occur at the heliospheric current sheet, is not possible. Rather, the turning of the flow patterns of open flux, outside of coronal holes, must occur outside the streamer-stalk region, as shown.

\subsection{Conclusions About the Behavior of the Heliospheric Magnetic Field}

Our basic proposition is that the width of the streamer-stalk region controls the magneticfield strength of the background level of the open magnetic flux in the region outside the streamer-stalk region. No other modification to models for the behavior of the open magnetic flux of the Sun that were developed in the previous solar minimum is required. However, there is still work to be done on how the magnetic field behaves in the streamer-stalk region. 
It is argued that it is a separate component from the magnetic field outside of the streamer stalk region, with different governing processes. The streamer-stalk region may contain the legs of ICMEs. It is certainly subject to disconnection at the current sheet.

\section{The Behavior of the Solar Wind}

The Fisk solar-wind acceleration model (Fisk, Schwadron, and Zurbuchen, 1998, 1999a; Fisk, 2003; Fisk and Zhao, 2009) is unlike the traditional solar-wind model (e.g., Parker (1958), Isenberg (1991) and references therein; Marsch (1995) and references therein; Hansteen and Leer (1995); Axford and McKenzie (1997); Cranmer, van Ballegooijen, and Edgar (2007)), in which there is a deposition of energy and perhaps momentum into the corona, and this deposition accelerates the solar wind and determines all other flow parameters, such as the solar-wind mass flux. In the Fisk solar-wind model, the mass flux is determined independently of the deposition of energy, by the release of material from coronal loops by reconnection.

In Fisk (2003), a solar-wind acceleration theory was developed in which the mass flux is determined by the release of material from coronal loops, and independent of the solar-wind acceleration mechanism. It is assumed that the plasma in the coronal loops is in hydrodynamic equilibrium at constant temperature; an integration of the density then gives the mass of the coronal loop that is contributed to the solar-wind mass flux:

$$
\rho_{i} u_{i} S_{i}=\left(\frac{\rho_{\mathrm{loop}, i}}{B_{\mathrm{loop}, i}}\right)\left(\frac{B_{\mathrm{open}, i} \cdot S_{i}}{\delta t}\right)\left(\frac{2 r_{0}^{2} k T}{G M_{0} m_{\mathrm{p}}}\right)\left[1-\exp \left(-\frac{1.75 h_{\mathrm{loop}} G M_{0} m_{\mathrm{p}}}{2 r_{0}^{2} K T}\right)\right],
$$

where $h_{\text {loop }}$ is the loop height; $\rho_{\text {loop }, i}$ is the mass density at the point of reconnection at the base of the loop; $G$ is the gravitational constant; $M_{0}$ is the mass of the Sun; $r_{0}$ is the solar radius; $m_{\mathrm{p}}$ is the mass of the proton, the dominant species; $T$ is the electron temperature of the coronal loop, which can be inferred from the solar-wind charge-state ratio by using the local thermal equilibrium model (Ko et al. 1997); $\delta t$ is the characteristic time for open field lines to execute their random displacements; $S_{i}$ is the total solar surface; and $k$ is the Boltzmann constant. The factor $\beta\left(h_{\text {loop }, i} T\right)=\left\{1-\exp \left[-1.75 h_{\text {loop }} G M_{0} m_{\mathrm{p}} /\left(2 r_{0}^{2} k T\right)\right]\right\}^{-1}$ corrects for the case where the height of the loop is comparable to or less than the scale height. The magnetic-field strength $\left[B_{\text {open }}\right]$ is the average open magnetic-field strength in this region of the corona. The factor of $\left(\rho_{\mathrm{loop}, i} / B_{\mathrm{loop}, i}\right)$ can be considered relatively constant (Fisk, 2003). Therefore, the mass released from a loop is roughly proportional to the electron temperature of the loop.

The deposition of energy into the corona is proportional to the magnetic energy contained in the flux tube along which the solar wind is being accelerated. By substituting the deposition energy and the solar-wind mass flux, given in Equation (1), into the standard MHD energy balance equation, Fisk (2003) finds that the final speed of the solar wind is given by

$$
\frac{u_{\mathrm{f}}^{2}}{2}=\left(\frac{B_{\text {loop }, i}}{\rho_{\text {loop }, i}}\right)\left(\frac{\int \mathbf{B}_{\text {open }} \cdot \mathrm{dh}}{4 \pi r_{0}}\right)\left(\frac{G M_{0} m_{\mathrm{p}}}{2 r_{0}^{2} k T}\right) \beta\left(h_{\text {loop }, T}\right)-\frac{G M_{0}}{r_{0}} .
$$

The anti-correlation between the solar-wind speed square $\left[u_{\mathrm{f}}^{2}\right]$ and the coronal electron temperature $[T]$ in Equation (2) has been confirmed by the Ulysses observations near the Cycle 22 -23 solar minimum (27 August 1996-9 February 1997) (Gloeckler, Zurbuchen, and Geiss, 2003). We will examine the final solar-wind mass flux Equation (1) and the final speed Equation (2) with the in-situ observations in the Cycle 23-24 minimum. 

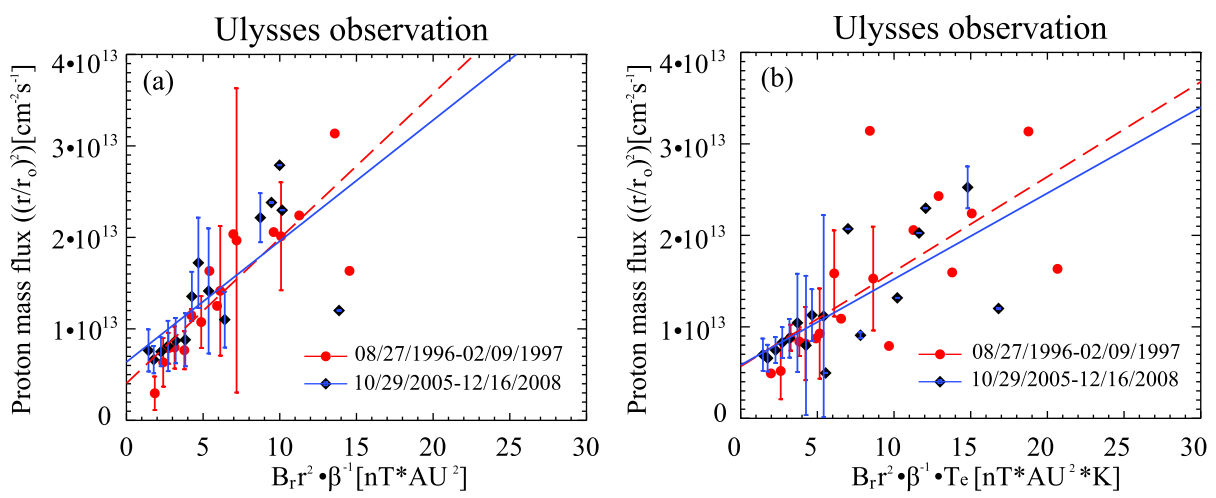

Figure 12 The mass flux of the solar wind versus the bin-averaged, normalized component of the heliospheric magnetic field, multiplied by the correction factor (a) and also by the electron temperature (b), for the same time periods as shown in Figure 6, from Ulysses observations. The binning technique is described in the text.

\subsection{The Mass Flux}

We note that, as derived in Fisk (2003), $\beta^{-1}, B_{r} r^{2}$, and $T$ enter into the formula of mass flux in Equation (1). Therefore, we expect that there may be a linear relationship between $\rho_{\mathrm{SW}} u_{\mathrm{SW}} S \sim B_{r} r^{2} \beta^{-1}$ or $\rho_{\mathrm{Sw}} u_{\mathrm{SW}} S \sim T B_{r} r^{2} \beta^{-1}$ in the in-situ Ulysses data. When calculating $\beta$, we use a simple assumption for the relation between the loop height and the electron temperature. Instead of assuming a linear relation as in Gloeckler, Zurbuchen, and Geiss (2003), we use an exponential curve to fit the temperature dependency of the loop heights and our assumption agrees well with the existing limited observations (Feldman, Widing, and Warren, 1999). In Figure 12, we present the relationship of $\rho_{\mathrm{SW}} u_{\mathrm{SW}} S \sim B_{r} r^{2} \beta^{-1}$ and $\rho_{\mathrm{SW}} u_{\mathrm{SW}} S \sim T B_{r} r^{2} \beta^{-1}$, respectively. In addition, we excluded ICMEs using the criterion in Table 1 (Zhao, Zurbuchen, and Fisk, 2009). To compare with the result of Gloeckler, Zurbuchen, and Geiss (2003) for the minimum of Cycle 22-23, we adopt the time period that they used in their article during that solar minimum, which is 27 August 1996 - 9 February 1997; and we use the same period for the Cycle 23-24 solar minimum as used in Figure 6. We first average the data in every 36-hour period. Then we average these 36 -hour-averaged values in every $B_{r} r^{2} \beta^{-1}=0.5$ (or $T B_{r} r^{2} \beta^{-1}=0.5$ ) bins along the $x$-axis, and present those bin-averaged values in solid-red circles (minimum of Cycle 22-23) and black diamonds (minimum of Cycle 23-24) in Figure 12. This process removes the variations in the mass flux that will be introduced by the other parameters in Equation (1) besides $T, B_{r} r^{2}$, or $\beta^{-1}$. The relationships in Figures 12a and 12b are linear, as predicted in Equation (1). If we exclude those binned data averaged from one single 36-hour-averaged data point, the linear coefficient will be as high as $\approx 0.9$. Note also that the intercepts in Figure 12 are close to 0 ; they are just about $10 \%$ of the total average value of the mass flux as shown in the figure. The strong linear relations in the minimum of Cycle 23-24 are consistent with the theoretical prediction and suggest that the entire mass flux is due to the processes described by Equation (1), as opposed to, e.g., a portion of the mass flux being due only to the acceleration process, as in a standard solar-wind model.

The correlation between $\rho_{\mathrm{Sw}} u_{\mathrm{SW}} S$ and $B_{r} r^{2}$ has also been reported in the minimum of Cycle 23-24 (27 February 2006-10 June 2007, and 18 October 2007-1 April 2008) (Schwadron and McComas, 2008). Without including the correction factor and electron tem- 

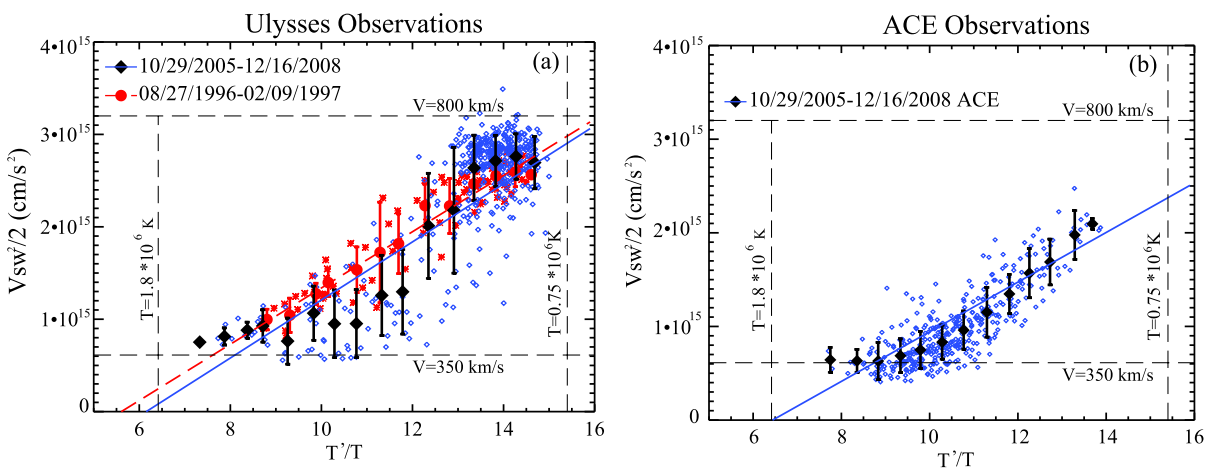

Figure 13 The anti-correlation between the solar-wind speed squared and the coronal electron temperature as measured by solar-wind charge states from Ulysses (a) and ACE (b) observation. Two time periods are shown. The first is the same as in the Gloeckler, Zurbuchen, and Geiss (2003) analysis; the second is for the minimum of Cycle $23-24$.

perature, they obtained a strong correlation; however, the intercept of their linear fitted line is not close to zero as predicted by Equation (1).

\subsection{The Solar Wind Flow Speed}

We calculate the electron temperature $[T]$ at the source of the solar wind using the local thermal equilibrium model provided by Ko et al. (1997). As discussed by Gloeckler, Zurbuchen, and Geiss (2003), by using this model, the typical measurement error of $T$ is $\approx 4.2 \%$ for $T \approx 1 \times 10^{6} \mathrm{~K}$ and $3.2 \%$ for $T \approx 1.7 \times 10^{6} \mathrm{~K}$. In Figure 13 we show the plots of $Y=V_{\mathrm{sw}}^{2} / 2$ versus $X=G M m \beta / 2 r_{s} k T=T^{\prime} / T$ in the Cycle $22-23$ solar minimum (27 August 1996 - 9 February 1997, Gloeckler, Zurbuchen, and Geiss, 2003) and the minimum of Cycle 23 - 24 (29 October 2005 - 16 December 2008, CR 2036-2077), excluding the periods of CMEs identified by the criteria in Table 1 (Zhao, Zurbuchen, and Fisk, 2009). The small data symbols shown in Figure 13 are the 36-hour-averaged values from the hourly observations, and the larger symbols indicate the bin-averaged value in each bin (bin size $\triangle X=0.5$ ). Clearly, there are strong linear relations in each of the solar minima, and in both Ulysses and ACE, as predicted by the solar-wind Equation (2), indicating that despite the obvious changes in the minimum of Cycle $23-24$, the solar-wind acceleration theory developed in the previous minimum of Cycle 22-23 (Fisk, Schwadron, and Zurbuchen, 1998, 1999a; Fisk, 2003; Fisk and Zhao, 2009) still holds.

The latest observations from ACE and Ulysses in the current, unusual solar minimum show that the basic formulae that were derived to explain the mass flux and anti-correlation between solar-wind speed and coronal electron temperature still hold, without alteration.

\section{Concluding Remarks}

The principal conclusions of this paper are that it is necessary to make only simple adjustments to previously developed models for the behavior of the open magnetic flux of the Sun and the solar wind to account for the important characteristics of the behavior of the heliospheric magnetic field and the solar wind in the current unusual solar minimum: 
- A streamer-stalk region that behaves separately from the region outside the streamer-stalk region can account for the reduction in the background magnetic field strength.

- The relationships for solar-wind mass flux and the solar-wind flow speed still hold in the minimum of Cycle $23-24$.

The minimum of Cycle 23-24 is different from the minimum of Cycle 22-23 as reported by many observations. However, the fact that the solar-wind acceleration model developed in the Cycle 22 - 23 solar minimum still holds in the minimum of Cycle 23-24 suggests that the fundamental physical process that accelerates the solar wind has not changed, and with small alterations the model for the behavior of the heliospheric magnetic field remains valid. In that sense, as far as the heliosphere is concerned, the minimum of Cycle $23-24$ is not particularly unusual.

It should be noted that we do not deal with the root cause of the variation in the width of the streamer-stalk region or the change in the parameters governing mass flux and speed, other than to note that the width and the parameters are consistent with a less-active Sun. Moreover, we clearly need to explore in more detail the behavior of the magnetic field in the streamer region, and its role in controlling the behavior of the magnetic open flux.

Acknowledgements This research was supported by the NASA Living With a Star Heliophysics Postdoctoral Fellowship Program, administered by the University Corporation for Atmospheric Research (NCAR), which is supported by the National Science Foundation. And this work has also been supported in part by NASA Earth and Space Science Fellowship Program-Grant NNX09AV13H, by the Heliophysics Theory Program, by NSF grant ATM0632471, and by grant NNX08AM64G. The work has also benefited greatly from the discussions in the solar and heliospheric research group at the University of Michigan; the discussions with Thomas Zurubuchen and Susan Lepri were especially helpful.

\section{References}

Abramenko, V., Yurchyshyn, V., Linker, J., Mikić, Z., Luhmann, J., Lee, C.O.: 2010, Lowlatitude coronal holes at the minimum of the 23rd solar cycle. Astrophys. J. 712, 813-818. doi: $10.1088 / 0004-637 X / 712 / 2 / 813$.

Axford, W.I., McKenzie, J.F.: 1997, The solar wind. In: Jokipii, J.R., Sonett, C.P., Giampapa, M.S. (eds.) Cosmic Winds and the Heliosphere, U. Arizona, Tucson, 31.

Balogh, A., Smith, E.J.: 2001, The heliospheric magnetic field at solar maximum: Ulysses observations. Space Sci. Rev. 97, 147 - 160. doi:10.1023/A:1011854901760.

Cranmer, S.R., van Ballegooijen, A.A., Edgar, R.J.: 2007, Self-consistent coronal heating and solar wind acceleration from anisotropic magnetohydrodynamic turbulence. Astrophys. J. Suppl. 171, 520-551. doi:10.1086/518001.

Crooker, N.U., Gosling, J.T., Kahler, S.W.: 2002, Reducing heliospheric magnetic flux from coronal mass ejections without disconnection. J. Geophys. Res. 107, 1028. doi:10.1029/2001JA000236.

Crooker, N.U., Burton, M.E., Siscoe, G.L., Kahler, S.W., Gosling, J.T., Smith, E.J.: 1996, Solar wind streamer belt structure. J. Geophys. Res. 101, 24331 - 24342. doi:10.1029/96JA02412.

de Toma, G.: 2010, Evolution of coronal holes and implications for high-speed solar wind during the minimum between Cycles 23 and 24. Solar Phys., 213-238. doi:10.1007/s11207-010-9677-2.

Feldman, U., Landi, E., Schwadron, N.A.: 2005, On the sources of fast and slow solar wind. J. Geophys. Res. 110, 7109. doi:10.1029/2004JA010918.

Feldman, U., Widing, K.G., Warren, H.P.: 1999, Morphology of the quiet solar upper atmosphere in the $4 \times 10^{4}<T_{\mathrm{e}}<1.4 \times 10^{6} \mathrm{~K}$ temperature regime. Astrophys. J. 522, $1133-1147$. doi:10.1086/307682.

Fisk, L.A.: 1996, Motion of the footpoints of heliospheric magnetic field lines at the Sun: Implications for recurrent energetic particle events at high heliographic latitudes. J. Geophys. Res. 101, 15547-15554. doi:10.1029/96JA01005.

Fisk, L.A.: 2003, Acceleration of the solar wind as a result of the reconnection of open magnetic flux with coronal loops. J. Geophys. Res. 108, 1157. doi:10.1029/2002JA009284.

Fisk, L.A.: 2005, The open magnetic flux of the Sun. I. Transport by reconnections with coronal loops. Astrophys. J. 626, $563-573$. doi:10.1086/429957. 
Fisk, L.A., Schwadron, N.A.: 2001, The behavior of the open magnetic field of the Sun. Astrophys. J. 560, 425 - 438. doi:10.1086/322503.

Fisk, L.A., Zhao, L.: 2009, The heliospheric magnetic field and the solar wind during the solar cycle. In: Gopalswamy, N., Webb, D.F. (eds.) Universal Heliospheric Processes, IAU Symp. 257, Cambridge Univ. Press, Cambridge, 109-120. doi:10.1017/S1743921309029160.

Fisk, L.A., Zurbuchen, T.H.: 2006, Distribution and properties of open magnetic flux outside of coronal holes. J. Geophys. Res. 111, 9115. doi:10.1029/2005JA011575.

Fisk, L.A., Schwadron, N.A., Zurbuchen, T.H.: 1998, On the slow solar wind. Space Sci. Rev. 86, 51 -60. doi:10.1023/A:1005015527146.

Fisk, L.A., Schwadron, N.A., Zurbuchen, T.H.: 1999a, Acceleration of the fast solar wind by the emergence of new magnetic flux. J. Geophys. Res. 104, 19765 - 19772. doi:10.1029/1999JA900256.

Fisk, L.A., Zurbuchen, T.H., Schwadron, N.A.: 1999b, On the coronal magnetic field: Consequences of largescale motions. Astrophys. J. 521, $868-877$. doi:10.1086/307556.

Geiss, J., Gloeckler, G., von Steiger, R.: 1995, Origin of the solar wind from composition data. Space Sci. Rev. 72, 49-60. doi:10.1007/BF00768753.

Gloeckler, G., Zurbuchen, T.H., Geiss, J.: 2003, Implications of the observed anticorrelation between solar wind speed and coronal electron temperature. J. Geophys. Res. 108, 1158. doi:10.1029/2002JA009286.

Gosling, J.T., Skoug, R.M.: 2002, On the origin of radial magnetic fields in the heliosphere. J. Geophys. Res. 107, 1327. doi:10.1029/2002JA009434.

Gosling, J.T., Birn, J., Hesse, M.: 1995, Three-dimensional magnetic reconnection and the magnetic topology of coronal mass ejection events. Geophys. Res. Lett. 22, 869-872. doi:10.1029/95GL00270.

Hansteen, V.H., Leer, E.: 1995, Coronal heating, densities, and temperatures and solar wind acceleration. J. Geophys. Res. 100, 21577 - 21594. doi:10.1029/95JA02300.

Isenberg, P.A.: 1991, Interstellar pickup protons at pressure-balanced structures. J. Geophys. Res. 96, $155-$ 159. doi:10.1029/90JA01994.

Jones, G.H., Balogh, A.: 2003, The global heliospheric magnetic field polarity distribution as seen at Ulysses. Ann. Geophys. 21, $1377-1382$.

Kirk, M.S., Pesnell, W.D., Young, C.A., Hess Webber, S.A.: 2009, Automated detection of EUV polar coronal holes during Solar Cycle 23. Solar Phys. 257, 99 - 112. doi:10.1007/s11207-009-9369-y.

Ko, Y., Fisk, L.A., Geiss, J., Gloeckler, G., Guhathakurta, M.: 1997, An empirical study of the electron temperature and heavy ion velocities in the south polar coronal hole. Solar Phys. 171, 345-361.

Kojima, M., Kakinuma, T.: 1987, Solar cycle evolution of solar wind speed structure between 1973 and 1985 observed with the interplanetary scintillation method. J. Geophys. Res. 92, 7269-7279. doi:10.1029/JA092iA07p07269.

Lin, R.P., Kahler, S.W.: 1992, Interplanetary magnetic field connection to the sun during electron heat flux dropouts in the solar wind. J. Geophys. Res. 97, 8203-8209. doi:10.1029/92JA00230.

Marsch, E.: 1995, The microscopic state of the solar wind: Links between composition, velocity distributions, waves and turbulence. In: Winterhalter, D., Gosling, J.T., Habbal, S.R., Hurth, W.S., Neugebauer, M. (eds.) Solar Wind Eight. Am. Inst. Phys., Woodbury, 35.

McComas, D.J., Ebert, R.W., Elliott, H.A., Goldstein, B.E., Gosling, J.T., Schwadron, N.A., Skoug, R.M.: 2008, Weaker solar wind from the polar coronal holes and the whole Sun. Geophys. Res. Lett. 35, 18103. doi:10.1029/2008GL034896.

Miyake, W., Mukai, T., Oyama, K., Terasawa, T., Hirao, K.: 1989, Thin equatorial low-speed region in the solar wind observed during the recent solar minimum. J. Geophys. Res. 94, 15359-15365. doi:10. 1029/JA094iA11p15359.

Neugebauer, M., Forsyth, R.J., Galvin, A.B., Harvey, K.L., Hoeksema, J.T., Lazarus, A.J., Lepping, R.P., Linker, J.A., Mikic, Z., Steinberg, J.T., von Steiger, R., Wang, Y., Wimmer-Schweingruber, R.F.: 1998, Spatial structure of the solar wind and comparisons with solar data and models. J. Geophys. Res. 103, 14587 - 14600. doi:10.1029/98JA00798.

Owens, M.J., Crooker, N.U., Schwadron, N.A., Horbury, T.S., Yashiro, S., Xie, H., St. Cyr, O.C., Gopalswamy, N.: 2008, Conservation of open solar magnetic flux and the floor in the heliospheric magnetic field. Geophys. Res. Lett. 35, 20108. doi:10.1029/2008GL035813.

Pagel, C., Crooker, N.U., Larson, D.E.: 2005, Assessing electron heat flux dropouts as signatures of magnetic field line disconnection from the Sun. Geophys. Res. Lett. 32, 14105. doi:10.1029/2005GL023043.

Parker, E.N.: 1958, Dynamics of the interplanetary gas and magnetic fields. Astrophys. J. $128,664$. doi:10.1086/146579.

Phillips, J.L., Bame, S.J., Barnes, A., Barraclough, B.L., Feldman, W.C., Goldstein, B.E., Gosling, J.T., Hoogeveen, G.W., McComas, D.J., Neugebauer, M., Suess, S.T.: 1995, Ulysses solar wind plasma observations from pole to pole. Geophys. Res. Lett. 22, 3301 - 3304. doi:10.1029/95GL03094.

Richardson, J.D., Paularena, K.I.: 1997, Streamer belt structure at solar minima. Geophys. Res. Lett. 24, 1435 - 1438. doi:10.1029/97GL01251. 
Schwadron, N.A., McComas, D.J.: 2003, Solar wind scaling law. Astrophys. J. 599, 1395 - 1403. doi:10.1086/ 379541.

Schwadron, N.A., McComas, D.J.: 2008, The solar wind power from magnetic flux. Astrophys. J. Lett. 686, L33 - L36. doi:10.1086/592877.

Schwadron, N.A., McComas, D.J., DeForest, C.: 2006, Relationship between solar wind and coronal heating: Scaling laws from solar X-Rays. Astrophys. J. 642, 1173 - 1176. doi:10.1086/501066.

Smith, E.J., Balogh, A.: 2008, Decrease in heliospheric magnetic flux in this solar minimum: Recent Ulysses magnetic field observations. Geophys. Res. Lett. 35, 22103. doi:10.1029/2008GL035345.

Smith, E.J., Balogh, A., Lepping, R.P., Neugebauer, M., Phillips, J., Tsurutani, B.T.: 1995, ULYSSES observations of latitude gradients in the heliospheric magnetic field. Adv. Space Res. 16, 165-170. doi:10.1016/0273-1177(95)00328-C.

Svalgaard, L., Cliver, E.W.: 2007, A floor in the solar wind magnetic field. Astrophys. J. Lett. 661, L203L206. doi:10.1086/518786.

Tokumaru, M., Kojima, M., Fujiki, K., Hayashi, K.: 2009, Non-dipolar solar wind structure observed in the Cycle 23/24 minimum. Geophys. Res. Lett. 36, 9101. doi:10.1029/2009GL037461.

von Steiger, R., Schwadron, N.A., Fisk, L.A., Geiss, J., Gloeckler, G., Hefti, S., Wilken, B., WimmerSchweingruber, R.F., Zurbuchen, T.H.: 2000, Composition of quasi-stationary solar wind flows from Ulysses/Solar Wind Ion Composition Spectrometer. J. Geophys. Res. 105, 27217-27238. doi:10.1029/1999JA000358.

Zhao, L., Fisk, L.: 2010, Comparison of two solar minima: Narrower streamer stalk region and conserved open magnetic flux in the region outside of streamer stalks. In: Cranmer, S.R., Hoeksema, J.T., Kohl, J.L. (eds.) CS-428, Astron. Soc. Pacific, San Francisco, 229.

Zhao, L., Zurbuchen, T.H., Fisk, L.A.: 2009, Global distribution of the solar wind during Solar Cycle 23: ACE observations. Geophys. Res. Lett. 36, 14104. doi:10.1029/2009GL039181.

Zurbuchen, T.H., Hefti, S., Fisk, L.A., Gloeckler, G., Schwadron, N.A.: 2000, Magnetic structure of the slow solar wind: Constraints from composition data. J. Geophys. Res. 105, 18327-18336. doi:10.1029/1999JA000427.

Zurbuchen, T.H., Fisk, L.A., Gloeckler, G., von Steiger, R.: 2002, The solar wind composition throughout the solar cycle: A continuum of dynamic states. Geophys. Res. Lett. 29(9), 090000. doi:10.1029/ 2001 GL013946. 